\title{
Experimental Study on the Neuronal Toxical Effect of Levodopa and the Inhibition of Ginkgo Biloba Extract on Parkinson Disease in Rats
}

\author{
Cao Fei*, Sun Sheng-Gang and Tong E-Tang
}

Department of Neurology, Union Hospital, Tong-ji Medical College, Hua-zhong Science and Technology University, Wuhan, PR China

\begin{abstract}
Objective: To observe neuronal toxical effect of Levodopa and investigate whether using Levodopa together with $\mathrm{EGb}$ is an ideal, workable method to treat Parkinson disease.

Methods: In this study, the rat models of Parkinson disease (PD) were made by injecting stereotaxically 6-OHDA to right side of the mesencephic ventral tegmental area (VTA) and substantia nigra pars compacta (SNc). We used rotational behaviour, TUNEL, immunocytochemical, Nissl's body staining methods to observe the difference between Levodopa (50mg/kg/d×3d, ×5d, ×7d, L-dopa group) and the combination use of Levodopa and EGb (100 mg/kg/d, E-D group)

Results: The numbers of apoptosis and rotation, bFGF protein expression in the L-dopa group surpassed those in the E-D group $(P<0.05)$. The number of Nissl cells in the L-dopa group was fewer than the E-D group.

Conclusion: Levodopa has neurological toxical effect. EGb may decrease the toxicity of levodopa. The combination use of Ginkgo biloba extract (EGb) and Levodopa is a workable method to treat Parkinson disease and is better than using Levodopa alone.
\end{abstract}

Keywords: Ginkgo biloba extract (EGb); Apoptosis; L-dihydroxyphenylalanine (L-dopa)

\section{Introduction}

The background of Parkinson disease is degeneration of neuronal degeneration and death of compact part in substantial nigra of mesencephalon, and decreasing release of dopamine in striatum. The etiology is not clear yet. On the aspect of therapy, traditional substitutive therapy of dopamine is majority, levedopa and its compound agent are major medication. Although having neuronal toxical effect of self-oxidation [1], levodopa cannot be substituted because of its reliable effects. Therefore the problem how to use levodopa reasonablely has not been solved. We should confront. The underlying medical effect of Ginkgo biloba extract (EGb) is anti- free radical and anti-palate active factor (PAF). Ginkgo biloba extract has been used to treat cerebrovascular disease because of its reliable effects. Ginkgo biloba extract. Still to now, it has not be reported that treating Parkinson disease using levodopa combining with Ginkgo biloba extract. Using these methods: TUNEL, praxeology, ultrastructural, immuno- histochemistry, we explore to identify if the treatment is feasible. The aim of our research is look for a new method to decrease the toxical effect of levodopa, increase the effect of levodopa.

\section{Materials and Methods}

\section{Animal}

Select healthy Wistar male rats old as three month, provided by the center of animal, Tong-ji Medical College, Hua-zhong Science and Technology University, weight 180-200 gram. Behaviour of each rat was checked to be normal.

\section{Drug}

6-hydroxydopamine (6-OHDA) parenteral solution to provide: 6-hydroxydopamine and Vitmine C shaf powder (Sigma company produce) according to the ratio of 2.5:1, solutes into Sodium chloride solutions, dispensing into $0.3 \%$ 6-OHDA solution. It should be used as soon as dispensed and preserved in hypothermia, avoiding light and enclosed.

\section{Ginkgo biloba extract (EGb) Suspension}

Material of EGb was offered by Kang Eng-Bei medical conglomerate, Zhe Jang province, China. EGb portion is: $24 \%$ flavone, $6 \%$ ginkgo lactone. The material of EGb was mixed with carboxymethylcellulose sodium to produce EGb Suspension. Concentration of EGb Suspension is $20 \mathrm{mg} / \mathrm{ml}$. It should be used as soon as dispensed and preserved in hypothermia, avoiding light and enclosed. Before being used, EGb Suspension should be agitated by BIM-8A ultrasound shoker.

\section{Levodopa parenteral solution}

Levodopa shaf powde (Sigma company produce) was dissolved into injection solution containing $0.05 \%$ ethanol and $0.1 \%$ ascorbic acid. Concentration of Levodopa parenteral solution is $50 \mathrm{mg} / \mathrm{ml}$. It should be used as soon as dispensed and preserved in hypothermia, avoiding light and enclosed.

\section{Reagents and equipments}

Apoptosis Test Kit (Boehringer Mannheim produce, German), $5 \%$ bamboo peony stain (Boster company produce, China), Anti-rat IgG bFGF 1:100 (Santa Cruz company produce, America). Animal

*Corresponding author: Cao Fei, Department of Neurology, Union Hospital, Tongji Medical College, Huazhong University of Science and Technology, Wuhan, PR China, E-mail: caofyx@163.com

\section{Received April 11, 2013; Accepted July 28, 2013; Published July 31, 2013}

Citation: Fei C, Sheng-Gang S, E-Tang T (2013) Experimental Study on the Neuronal Toxical Effect of Levodopa and the Inhibition of Ginkgo Biloba Extract on Parkinson Disease in Rats. J Neurol Disord 2: 126. doi:10.4172/23296895.1000126

Copyright: (C) 2013 Fei C, et al. This is an open-access article distributed under the terms of the Creative Commons Attribution License, which permits unrestricted use, distribution, and reproduction in any medium, provided the original author and source are credited. 
brain solid positioner (Xi Bei optic instrument factory, China), media mix color pathologic analysis system (Computer Center of Beijing aerospace college produce).

\section{Animal model making}

Following the method of Thomas [2], we made uni-lateral 6-hydrodopamine-lesioned Parkinson disease rats model. The site of locus substantial nigra compact part $(\mathrm{SNc})$ and mid-brain ventral tegmental area (VTA) was determined previously. The needle was inserted at the following coordinates with respect to lambda and dura. The coordinate of SNc is: anterior fontanelle posterior (AP) $4.8 \mathrm{~mm}$, Media line Right-Sidedness(ML) $2.0 \mathrm{~mm}$, dura mater vertical(DV) 7.5 $\mathrm{mm}$; The coordinate of VTA is AP4.4 mm, ML1.2 mm, DV7.5 mm. Each of the two injection contained 6-OHDA parenteral solution $3.3 \mu \mathrm{l}$. The neurotoxin was injected slowly at $1.0 \mu \mathrm{l} / \mathrm{min}$ and then the needle was left in place for 2-3 min after each injection. The syringe was then withdrawn at $1.0 \mathrm{~mm}$ duration and the skin closed.

\section{Behaviour testing}

One week following the lesion, the rats were injected abdominally with Apomophine (APO) $0.5 \mathrm{mg} / \mathrm{kg}$ to evoke rotational behaviour, and those rats which rotation were more than $210 \mathrm{ring} / 30 \mathrm{~min}$ were thought to be succeed PD rats model.

\section{PD rats groups and administration}

Forty-five succeed PD rats model were selective, divided into three groups randomly: control group ( $n=15)$, Levodopa group ( $n=15)$, EGbLevodopa therapeutic alliance group (E-D group, $n=15)$. 10 daya, 12 days, 14 days was observative time point. Each group had five rats at each time point. In E-D group, PD rats were perfused stomach with EGb suspension $100 \mathrm{mg} / \mathrm{kg} / \mathrm{d}$ for seven days, in the seventh day rats were injected abdominally with levodopa parenteral solution $50 \mathrm{mg} /$ $\mathrm{kg} / \mathrm{d}$, till to the the terminal time point. Levodopa group were perfused stomach with the same dose of solvent of EGb suspension for seven days, in the seventh day rats were injected abdominally with levodopa parenteral solution $50 \mathrm{mg} / \mathrm{kg} / \mathrm{d}$, till to the the terminal time point. The control group were perfused stomach with solvent of EGb suspension for seven days, in the seventh day rats were injected abdominally with the same dose of solvent of levodopa parenteral solution, till to the terminal time point.

\section{Experimental Marker}

\section{Praxeology observing}

Rotational behaviour of all groups was observed in the day after reservation time point. Each $\mathrm{PD}$ rat was injected apomophine 0.5 $\mathrm{mg} / \mathrm{kg}$ abdominally, and observed continuously two hours. After observation, rats were instillated and fixed with $4 \%$ polymerisatum perfusate with through penetrating heart and then break head select mid-brain, embedding with paraffin wax.

\section{Method of apoptosis detecting}

Each paraffin tissue mass section was $5 \mathrm{pm}$ thick. According to Seaton's method of apoptosis detecting [3].

\section{Nissl's staining of substantial nigra neuron}

Each paraffin tissue mass section was $5 \mathrm{pm}$ thick and stained for 1 to 2 minutes with bamboo peony aqueous solution and then washing, 95\% alcohol dischrom for 1 to 2 minutes, desiccation, clearing and sealing. At last each section was observed with microscope.

\section{Analysis of Image and Statistical Method}

Use media mix colorful pathological diagram analyse system to analyse diagram. Each section of apoptosis, immune-histochemistry, Nissl's staining was close neighbour. Measuring window was 262144 $\mu \mathrm{m}^{2}$. All data was indicated by the manner of mean \pm standard deviation, hypothesis testing; significance test of infra-group and intra-group was carried by Oxstat statistical program.

\section{Results}

\section{Apoptosis result of substantia nigra}

From Table 1, we can find that the apoptosis number of substantial nigra in E-D group decreased 33.4\%, 40.8\%, 35.7\% comparing with L-dopa group. And the difference was significant. The apoptosis number of substantial nigra in L-dopa group was $10 \mathrm{~d}<14 \mathrm{~d}<12 \mathrm{~d}$ (Table $1)$.

\section{Result of praxeology of rats}

Rats rotated toward uninjured side obeying the hour-hand of a clock, head connecting tail, taking hindlimb as fulcrum. The average time of starting was five to eight minutes, cost ten minutes to reach the highest rotation behaviour. The velocity of rotation was fifteen to eighteen. Interval of rotation behaviour was equal. Difference was significant. But there was no difference between E-D group with control group. The average time of starting and the time reaching highest rotation velocity were three to five minutes later than L-dopa group. The highest rotational velocity was seven to ten $\mathrm{ring} / \mathrm{min}$; Interval of rotation behaviour was different. In $10 \mathrm{~d}, 12 \mathrm{~d}, 14 \mathrm{~d}$, the mean rotation velocity decreased $31.4 \%$, 34.7\%, 32\% comparing with L-dopa group. The number of rotation in L-dopa group was $10 \mathrm{~d}<12 \mathrm{~d}<14 \mathrm{~d}$.

\section{Result of Nissl's neurons of substantial nigra}

The number of Nissl's neurons of substantial nigra in E-D group was higher than L-dopa group at each time point. The difference is significant. The number of Nissl's neurons in L-dopa group was $10 \mathrm{~d}<12 \mathrm{~d}<14 \mathrm{~d}$ (Table 2).

\section{Discussion}

\section{Neuronal toxical effect of L-dopa in substantial nigra}

Neurons in substantial nigra of patients with PD are easy to be attacked by free radical [3]. Melamed [4] think that neuronal toxical

\begin{tabular}{|l|c|c|c|c|}
\hline \multirow{2}{*}{ Group } & \multirow{2}{*}{ Number of rats } & \multicolumn{3}{|c|}{ Number of apoptosis (piece/mm ${ }^{2}$ ) } \\
\cline { 3 - 5 } & & $\mathbf{1 0 d}$ & $\mathbf{1 2 d}$ & $\mathbf{1 4 d}$ \\
\hline Control & 15 & $400 \pm 29$ & $388 \pm 26$ & $368 \pm 20$ \\
\hline L-dopa & 15 & $533 \pm 39 \triangle$ & $690 \pm 41^{\triangle}$ & $605 \pm 37 \triangle$ \\
\hline E-D & 15 & $355 \pm 26 * \triangle$ & $402 \pm 37^{\star}$ & $379 \pm 26 \%$ \\
\hline
\end{tabular}

Note: Comparing with L-dopa group, $※ \mathrm{P}<0.05$;

Comparing with the contral group, $\triangle \mathrm{P}<0.05$

Table 1: The apoptosis number of substantial nigra of rats at each time perfusing stomach $(X \pm S)$.

\begin{tabular}{|l|c|c|c|c|}
\hline \multirow{2}{*}{ Group } & \multirow{2}{*}{ Number of rats } & \multicolumn{3}{|c|}{ Rotation behavior (ring/30min) } \\
\cline { 3 - 5 } & & $\mathbf{1 0 d}$ & $\mathbf{1 2 d}$ & $\mathbf{1 4 d}$ \\
\hline Control & 15 & $231 \pm 22$ & $219 \pm 21$ & $220 \pm 19$ \\
\hline L-dopa & 15 & $318 \pm 19^{\triangle}$ & $340 \pm 23^{\triangle}$ & $360 \pm 20^{\triangle}$ \\
\hline E-D & 15 & $218 \pm 10^{\star}$ & $222 \pm 11^{\star}$ & $244 \pm 10^{*}$ \\
\hline
\end{tabular}

Note: Comparing with L-dopa group, $※ \mathrm{P}<0.05$;

Comparing with the contral group, $\triangle \mathrm{P}<0.05$

Table 2: The rotation behaviour of rats at each time perfusing stomach $(X \pm S)$. 
Citation: Fei C, Sheng-Gang S, E-Tang T (2013) Experimental Study on the Neuronal Toxical Effect of Levodopa and the Inhibition of Ginkgo Biloba Extract on Parkinson Disease in Rats. J Neurol Disord 2: 126. doi:10.4172/2329-6895.1000126

Page 3 of 3

\begin{tabular}{|l|c|c|c|c|}
\hline \multirow{2}{*}{ Group } & \multirow{2}{*}{ Number of rats } & \multicolumn{3}{|c|}{ Rotation behavior (ring/30min) } \\
\cline { 2 - 5 } & & $\mathbf{1 0 d}$ & $\mathbf{1 2 d}$ & 14d \\
\hline Control & 15 & $1750 \pm 211$ & $1695 \pm 207$ & $1499 \pm 188$ \\
\hline L-dopa & 15 & $1371 \pm 204 \triangle$ & $917 \pm 167 \triangle$ & $805 \pm 196 \triangle$ \\
\hline E-D & 15 & $1814 \pm 220 *$ & $1601 \pm 200 \%$ & $1303 \pm 172 \%$ \\
\hline
\end{tabular}

Note: Comparing with L-dopa group, ※P<0.05;

Comparing with the contral group, $\triangle \mathrm{P}<0.05$

Table 3: The number of Nissl,s neurons of substantial nigra at each time perfusing stomach $(\mathrm{X} \pm \mathrm{S})$

effect of L-dopa in substantial nigra is mainly caused by self-oxidizing. And the toxical effect leads to $\mathrm{PCl}_{2}$ neuron apoptosis and necrosis.

Mitochondria diplo-chain DNA (mtDNA) in nuclei is major object attacked by free radical. In our experiment, we find that number of apoptosis in substantial nigra increased with the time of L-dopa using. In $14 \mathrm{~d}$, because of the increasing of L-dopa neuronal toxical effect, dead pattern of neurons of substantial nigra changed from apoptosis pattern to necrosis pattern [5]. This opinion is the same as Mayo [6]. Nissl body is mark of stock cell. We found that the number of Nissl neuron was lower notably than the contral group. The finding, Nissl dody lysis from center or extinction, proved that neuronal toxical effect of L-dopa could make the function decreased or necrosis from $1 \mathrm{~d}$ to $21 \mathrm{~d}$.

\section{Mechanism of EGb inhibiting neuronal toxical effect of L- dopa}

Oxidative stress plays an important role in neuronal toxical effect of L-dopa. EGb effective background is cleaning free radical and anti-platelet active factor (PAF). Flavonoid glycoside has phenol hydroxy terrapin peroxidase prosthetic group $\mathrm{Fe}^{3+}$, inhibite its activity [7], inhibiting produce of free radical. EGb can depress the toxical effect of MPTP [8]. EGb can be inhibite Protease C, decrease velocity of apoptosis [9]. EGb and ginkgo lactone can affect function of dopaminergic system by means of membrane effect of platelet active factor. EGb and ginkgo lactone can inhibit dopaminergic metabolism of striatum and limbic system [10]. EGb prevents not only injury of mitochondria DNA, but also mitochondria function and morphologic change [11]. In our study, we found that number of apoptosis in E-D group was lower 33.4\%, 40.8\%, 35.7\% than L-dopa group in 10d, $12 \mathrm{~d}$, 14d. Number of Nissl's neurons in E-D group was higher than L-dopa group. And difference was significant. What said above states that $\mathrm{EGb}$ has neuronal protection of PD rats.

Rotation rings of rats are often used as a marker to reflect degree of dopaminergic neurons [12]. In the respect of praxeology, rotation rings of E-D group are lower notably than rats in L-dopa group. Testimony of praxeology states that EGb has neuronal protection of PD rats. Effect of therapeutic alliance of EGb and L-dopa is better than using L-dopa alone and neuronal toxical effect of L-dopa was well controlled.

To sum up, we learn that EGb can well control neuronal toxical effect of L-dopa and enlarge the range of L-dopa using safely [13]. EGb has little side effect. Therapeutic alliance of EGb and L-dopa will be useful in the treatment of PD.

\section{Acknowledgment}

This project was supported by grant from the National Nature Science Foundation of China.

\section{References}

1. Trugman JM, Hubbard CA, Bennett JP Jr (1996) Dose-related effects of continuous levodopa infusion in rats with unilateral lesions of the substantia nigra. Brain Res 725: 177-183.

2. Thomas J, Wang J, Takubo H, Sheng J, de Jesus S, et al. (1994) A 6-hydroxydopamine-induced selective parkinsonian rat model: further biochemical and behavioral characterization. Exp Neurol 126: 159-167.

3. Seaton TA, Cooper JM, Schapira AH (1998) Cyclosporin inhibition of apoptosis induced by mitochondrial complex I toxins. Brain Res 809: 12-17.

4. Melamed E, Offen D, Shirvan A, Djaldetti R, Barzilai A, et al. (1998) Levodopa toxicity and apoptosis. Ann Neurol 44: S149-154.

5. Jenner PG, Brin MF (1998) Levodopa neurotoxicity: experimental studies versus clinical relevance. Neurology 50: S39-43.

6. Mayo JC, Sainz RM, Antolín I, Rodriguez C (1999) Ultrastructural confirmation of neuronal protection by melatonin against the neurotoxin 6-hydroxydopamine cell damage. Brain Res 818: 221-227.

7. Ni Y, Zhao B, Hou J, Xin W (1996) Preventive effect of Ginkgo biloba extract on apoptosis in rat cerebellar neuronal cells induced by hydroxyl radicals. Neurosci Lett 214: 115-118.

8. Liu LL,Yu XR (1994) Value of ginkgo. Chinese herbal medicine 25: 219.

9. Xie PS (1998) Standard extract of ginkgo leaf EGb761and evaluation of ginkgo leaf agent mass. The traditional Chinese medicine 24: 3.

10. Wu C,You S, Liu W (1995) Influence of Ginkgo lactone and ginkgo leaf extracton content of dopamine and metabolite in striatum and limbic system. Chinese herbal medicine 26: 53 .

11. Sastre J, Pallardó FV, García de la Asunción J, Viña J (2000) Mitochondria, oxidative stress and aging. Free Radic Res 32: 189-198.

12. Tian X, Guan X, Liu B (1997) Influence of ganglioside on rotational behavior concentration of dopamine, pathology of substantia nigra. China Department of Neurology 30: 208.

13. Mattson MP, Cheng B (1993) Growth factors protect neurons against excitotoxic/ischemic damage by stabilizing calcium homeostasis. Stroke 24 I136-140. 